\title{
Changes in Heart Rate Variability in a Premature Infant with Hydrocephalus
}

\author{
Zuzana Uhrikova, M.D. ${ }^{1}$ Branislav Kolarovszki, M.D., Ph.D. ${ }^{3}$ Kamil Javorka, M.D., C.Sc. ${ }^{2}$ \\ Michal Javorka, M.D., Ph.D. ${ }^{2}$ Katarina Matasova, M.D., Ph.D. ${ }^{1}$ Hana Kolarovszka, M.D., Ph.D. ${ }^{1}$ \\ Mirko Zibolen, M.D., C.Sc. ${ }^{1}$
}

${ }^{1}$ Clinic of Neonatology, Jessenius Medical Faculty, Comenius

University, Martin, Slovakia Republic

2 Department of Physiology, Jessenius Medical Faculty, Comenius

University, Martin, Slovakia Republic

${ }^{3}$ Clinic of Neurosurgery, Jessenius Medical Faculty, Comenius

University, Martin, Slovakia Republic

Address for correspondence and reprint requests Branislav Kolarovszki, M.D., Ph.D., Clinic of Neurosurgery, Jessenius Medical Faculty, Comenius University, Kollarova 2, 03601 Martin, Slovak Republic (e-mail: kolarovszki@jfmed.uniba.sk).

Am J Perinatol Rep 2012;2:43-46.

\begin{abstract}
Keywords

- heart rate variability

- newborn

- hydrocephalus
\end{abstract}

Hydrocephalus is the abnormal enlargement of cerebral ventricles that results from impaired cerebrospinal fluid secretion, circulation, or resorption. Accumulation of cerebrospinal fluid in the intracranial space can cause an increase of the intracranial pressure, affecting central nervous system morphology and function. 1,2

Heart rate (HR) and heart rate variability (HRV) reflect alterations of the central cardiovascular control. Recently, evaluation of the short-term HRV by time domain analysis and spectral analysis using fast Fourier transformation has successfully been used to estimate cardiac autonomic regulation in newborns. Spectral frequency analysis yields information about the amount of variance (power) in the HR rhythm characterized by periodic oscillations of RR intervals (beat to beat) at various frequencies, which are regulated by a different manner through the autonomic nervous system. Spectral received

November 7, 2011

accepted after revision

February 23, 2012

published online

June 27, 2012
Copyright (c) 2012 by Thieme Medical Publishers, Inc., 333 Seventh Avenue, New York, NY 10001, USA. Tel: +1(212) 584-4662.
DOI http://dx.doi.org/ 10.1055/s-0032-1316462. ISSN 2157-6998. 
power in the low-frequency band (LF; 0.04 to $0.15 \mathrm{~Hz}$ ) is determined by both sympathetic and parasympathetic activity; power spectrum at $0.1 \mathrm{~Hz}$ reflects mainly baroreflex activity. Spectral power in the high-frequency band (HF; 0.15 to $0.5 \mathrm{~Hz}$ ) is dominantly influenced by parasympathetic vagal activity (respiratory sinus arrhythmia). Parameters of the time domain analysis SDRR (standard deviation of RR interval), SDRR\% (SDRR in percentage), and MSSD (mean squared successive differences between RR intervals in milliseconds squared) reflect mainly the magnitude of $R R$ interval oscillations. ${ }^{3,4}$

We hypothesize that HRV can be reduced in preterm infants with hydrocephalus, increased intracranial pressure, altered cerebral circulation, and decreased intracranial pressure by cerebrospinal fluid drainage, which can improve the central cardiovascular control. Therefore, the purpose of this work was to find and describe differences in HRV parameters in a preterm infant with hydrocephalus before and after the drainage procedure.

\section{Materials and Methods}

The premature female infant (birth weight $1480 \mathrm{~g}$, length 38 $\mathrm{cm}$ ) was born at gestational week 28 by means of cesarean section with Apgar scores of 3 and 6 points at 1 and 5 minutes. The pregnancy was complicated by preterm rupture of membranes and chorioamnionitis. After birth, resuscitation with prophylactic administration of surfactant was needed, and conventional mechanical ventilation was continued for the first 4 days. Early onset sepsis was confirmed by positive blood culture (Escherichia coli), and antibiotic treatment (ampicillin, gentamicin, and cefotaxime) was begun. Ultrasound examination of the brain at the age of 2 hours revealed germinal matrix hemorrhage. Repeated examinations during the first week did not show progression of hemorrhage. The infant was hemodynamically stable with respiratory support via nasal continuous positive airway pressure. At the age of 20 days, Doppler ultrasound examination of the brain confirmed the presence of four-ventricular hydrocephalus with impaired cerebral blood flow. The end-diastolic blood flow velocity (EDV) in the pericallosal artery was absent, and during the compression test on the anterior fontanelle, negative values were recorded, reflecting the reversed cerebral blood flow. Because of the presence of clinical signs of intracranial hypertension (full anterior fontanelle, diastasis of sagittal and lambdoid suture, increased head circumference [33 cm]), ultrasound and computed tomography findings of hydrocephalus, and alteration of cerebral circulation confirmed by Doppler ultrasound, a drainage operation was indicated. There was presented progression in the full anterior fontanelle, but no rapid neurological deterioration of the infant. Apnea and bradycardia were not detected after surgery. Prior to surgery, at the age of 20 days, ventricular puncture was performed to evaluate the cerebrospinal fluid and to choose the optimal type of drainage procedure. Direct measurement of intracranial pressure during the ventricular puncture confirmed intracranial hypertension $(17 \mathrm{~cm}$ of $\mathrm{H}_{2} \mathrm{O}$ ). The cerebrospinal fluid was clear, with normal protein content, and the ventriculoperitoneal $(\mathrm{V})$ shunt was inserted at the age of 22 days. During the drainage procedure, performed under general anesthesia, the infant was hemodynamically stable (mean arterial pressure [MAP] was 28 to $32 \mathrm{~mm} \mathrm{Hg}$ ), blood oxygen levels were normal, body temperature was 36 to $37^{\circ} \mathrm{C}$, and the following anesthetic drugs were used: sevoflurane, sufentanil, midazolam. Preoperative intracranial pressure was $15 \mathrm{~cm} \mathrm{H}_{2} \mathrm{O}$. After surgery, there were no signs of intracranial hypertension (normal finding on the anterior fontanelle, postoperatively initially slightly decreased $[32.5 \mathrm{~cm}]$ and subsequently stable head circumference), and ultrasound confirmed decrease in size of the cerebral ventricles and improvement of the cerebral circulation (increased EDV, decreased resistive index of the pericallosal artery). The following course was uneventful. The infant was discharged at the age of 71 days. The parameters of ultrasound examination before and after drainage operation and at discharge from the hospital are shown in - Table $\mathbf{1}$.

Three subsequent recordings of the electrocardiography (ECG) and HRV were done: the first at the age of 22 days before the insertion of VP shunt; the second at the age of 36 days with functional VP shunt; the third at the age of 71 days, before discharge. MAP was measured at the time of ECG recording, and values were normal: 35 to $38 \mathrm{~mm} \mathrm{Hg}$ (at the age of 22 days before the insertion of VP shunt), 40 to $42 \mathrm{~mm} \mathrm{Hg}$ (at the age of 36 days with functional VP shunt), and 45 to $50 \mathrm{~mm} \mathrm{Hg}$ (at the age of 71 days before discharge). The VarCor PF 6 Neonatal system (DIMEA, Olomouc, Czech Republic) was used for continuous recordings of ECG and RR intervals at sampling frequency of $1000 \mathrm{~Hz}$, using a bipolar thoracic ECG leads. For HRV analysis, a minimum of 4000 beats for each recording (30 minutes) were used. Data were filtered automatically by the elimination of artifacts using a recognition algorithm; they were also scanned visually and filtered manually. RR intervals were processed by the special software for spectral (fast Fourier transform) and time domain analysis of HRV. Wolfberg et al found that the correlation between the computer algorithm's assessment of HRV and the perinatologists' assessment was similar to the interrater reliability between perinatologists. ${ }^{5}$ Our results of the HRV spectral and time-domain analysis in a premature infant with hydrocephalus are shown in the - Table 1 and - Fig. 1.

\section{Discussion}

The accumulation of cerebrospinal fluid with dilatation of the cerebral ventricles in progressive neonatal hydrocephalus results in increased intracranial pressure with alteration of cerebral circulation and brain functions. ${ }^{6}$ When the cerebral blood flow, including the brain stem, is reduced, the compensatory cardiovascular response (Cushing reflex) mediated through the autonomic nervous system is started-bradycardia, arterial hypertension, and redistribution of blood flow. Therefore, we hypothesized that in the infant with increased intracranial pressure and altered cerebral circulation, the HRV reflecting chronotropic cardiac regulation could be reduced, and a decrease in the intracranial pressure by drainage procedure could improve the chronotropic cardiac regulation and parameters of HRV. 
Table 1 Parameters of HRV and Ultrasound Examinations in the Premature Infant with Hydrocephalus

\begin{tabular}{|c|c|c|c|}
\hline & Before Drainage & With Functional Ventriculoperitoneal Shunt & At Discharge \\
\hline \multicolumn{4}{|l|}{ Ultrasound examination } \\
\hline PSV $(\mathrm{cm} / \mathrm{s})$ & 31 & 33.3 & 36.4 \\
\hline $\mathrm{EDV}(\mathrm{cm} / \mathrm{s})$ & 0 & 8.4 & 8.9 \\
\hline RI & 1.00 & 0.79 & 0.75 \\
\hline $\mathrm{PI}$ & 1.93 & 1.33 & 1.38 \\
\hline $\mathrm{VI}(\mathrm{mm})$ & 32 & 22 & 18 \\
\hline $\mathrm{d}$ right $(\mathrm{mm})$ & 13 & 4 & 2.7 \\
\hline $\mathrm{d}$ left $(\mathrm{mm})$ & 13 & 4 & 3 \\
\hline $\mathrm{d}$ III (mm) & 7 & 3 & 3 \\
\hline \multicolumn{4}{|l|}{ Heart rate variability } \\
\hline Heart rate (beats per min) & 191 & 173 & 147 \\
\hline RR interval (s) & 0.314 & 0.346 & 0.409 \\
\hline SDRR (\%) & 0.014 & 0.02 & 0.041 \\
\hline SDRR (\%) & 4.43 & 5.81 & 10.04 \\
\hline $\operatorname{MSSD}\left(\mathrm{ms}^{2}\right)$ & 82.3 & 13.6 & 112 \\
\hline Total power $\left(\mathrm{ms}^{2}\right)$ & 7.38 & 50.7 & 252 \\
\hline Power LF $\left(\mathrm{ms}^{2}\right)$ & 6.1 & 45.8 & 191.3 \\
\hline Power HF $\left(\mathrm{ms}^{2}\right)$ & 1.25 & 4.9 & 61 \\
\hline Ratio HF/LF & 5.13 & 9.42 & 3.16 \\
\hline PSD LF $\left(\mathrm{ms}^{2} / \mathrm{Hz}\right)$ & 134 & 1168 & 4293 \\
\hline PSD HF $\left(\mathrm{ms}^{2} / \mathrm{Hz}\right)$ & 18 & 71 & 1154 \\
\hline Relative power LF (\%) & 83 & 90.4 & 75.8 \\
\hline Relative power HF (\%) & 17 & 9.6 & 24.2 \\
\hline
\end{tabular}

$\mathrm{d}$, width of the right and left lateral ventricle; $\mathrm{d}$ III, width of the third ventricle; EDV, end-diastolic flow velocity; HRV, heart rate variability; ICP, intracranial hypertension; MSSD, mean squared successive differences between RR intervals; PSD, power spectral density; PSV, peak systolic flow velocity; RI, resistive index; SDRR, standard deviation of the RR interval; SDRR \%, standard deviations of the RR interval; VI, ventricular index (distance between the frontal horns of lateral ventricles in the coronal view).

There are few studies about the alteration of HRV in relation to intracranial pressure changes in preterm infants. In a physiological study, Javorka et al found that the application of pressure on the anterior fontanelle evokes a significant decrease in the HR and inhibition of breathing, similar to the application of pressure on eye bulbs (oculocardiac reflex) in premature newborns. ${ }^{7}$ In adult patients, Van Ravenswaaij-Arts et al found a transient reduction in the HRV in cerebral edema. ${ }^{3}$ Robles et al showed autonomic nervous system dysfunction in $63 \%$ of newborns with hydrocephalus based upon reduced HRV. However, they did not study the effect of the cerebrospinal fluid drainage procedure. $^{8}$

In our patient, the cranial ultrasound examination before surgery showed signs of progressive hydrocephalus with dilatation of cerebral ventricles, decreased end-diastolic blood flow velocity, and increased pericallosal artery resistive index, reflecting impaired cerebral blood flow. Regarding HRV, there were low values of HRV in time as well as spectral domains. Sympathetic activity was dominant (ratio LF/HF and relative power LF, HF) accompanied by tachycardia.
After the drainage operation, with a functional VP shunt, ultrasound examination showed decreased cerebral ventricles and improved cerebral circulation. There was a significant increase in HRV parameters, particularly in the parameters of spectral analysis (total power and spectral powers in both LF and HF bands). Dominant sympathetic activity persisted, as seen in ratio LF/HF and relative power LF parameters, which were augmented.

The evaluation of HRV before discharge from the hospital showed decreased mean HR (prolongation of mean RR interval) and a great rise in HRV parameters in time (SDRR, SDRR\%), as well as spectral domain. Ratio $\mathrm{LF} / \mathrm{HF}$ and relative power $\mathrm{LF}$ decreased at the expense of relative power HF, reflecting enhanced parasympathetic activity.

Results of HRV analyses in a preterm infant with hydrocephalus before and after a drainage procedure demonstrated marked improvement in the chronotropic cardiac regulation after successful drainage. However, the findings have some limitations. We cannot compare the absolute data of the HRV parameters because there is a lack of relevant HRV reference values for preterm infants. The increase in all HRV spectral 

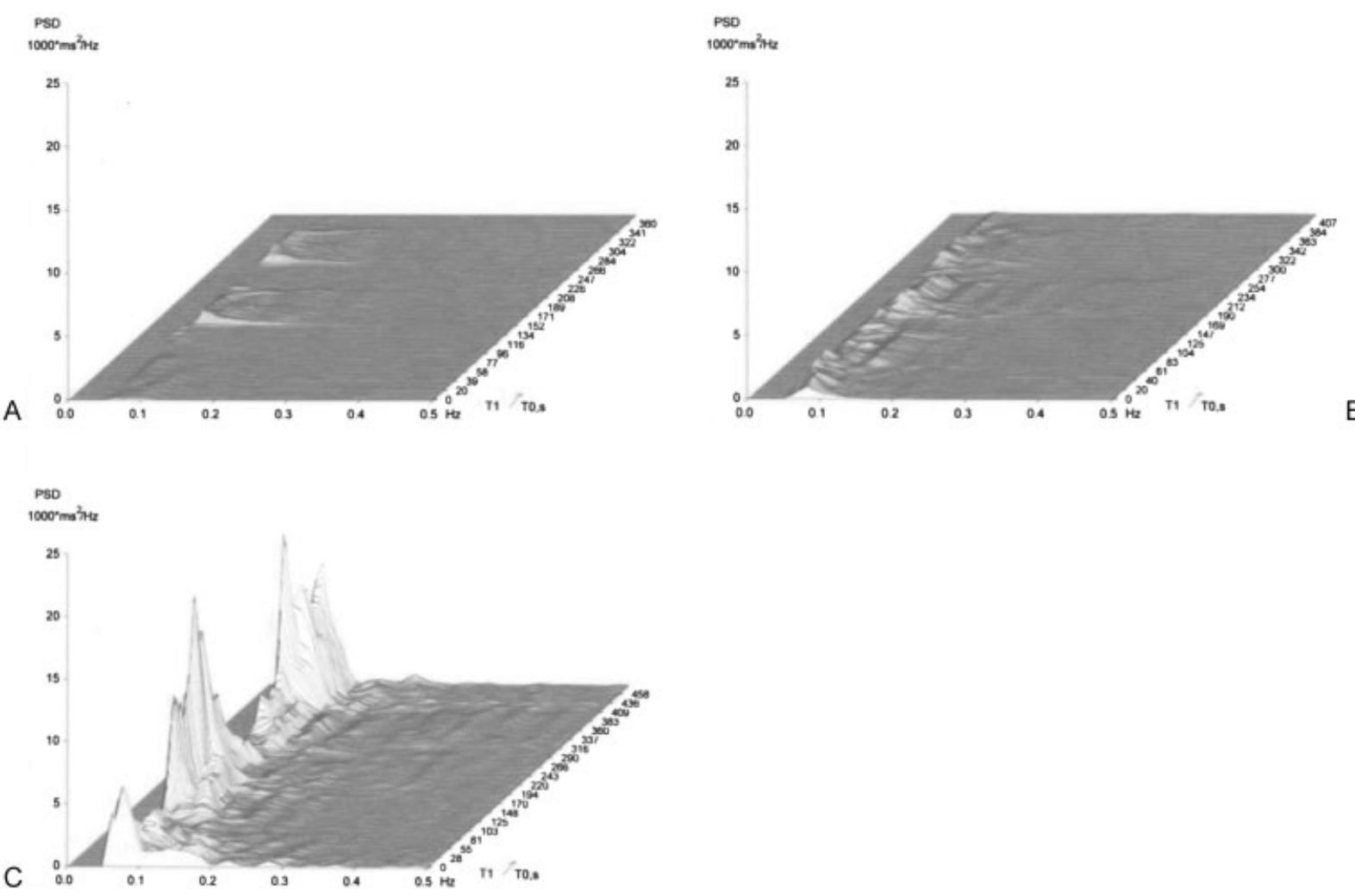

Figure 1 Three-dimensional running spectra (X-frequency in Hertz, Y-PSD, Z-time) examinations in the premature infant with hydrocephalus before the drainage procedure (A), with functional ventriculoperitoneal shunt (B), and before discharge from the hospital (C). PSD, power spectral densities in low- and high-frequency bands $\left(\mathrm{ms}^{2} / \mathrm{Hz}\right)$; $\mathrm{T}$, time.

power can be interpreted as the effect of successful drainage procedure with the decrease in intracranial pressure and the maturation of autonomic nervous system regulatory mechanisms. Age-dependent maturation of the regulation may be reflected mainly in enhanced parasympathetic cardiac regulation before discharging to home. Several studies confirmed that the maturation of the autonomic nervous system in preterm infants is accompanied by increasing HRV with a pronounced increase of parasympathetic activity. ${ }^{9,10}$

In conclusion, evaluation of HRV in a premature infants with hydrocephalus can be an additional method for monitoring cardiac dysregulation and improvement of the cardiovascular control after successful drainage procedure. Further studies in this field are required.

\section{Acknowledgments}

This work was supported by project Center of Excellence of Perinatology Research (CEPV II)", ITMS code: 26220120036, which is cofinanced by EU sources and by VEGA N.1/ $0073 / 09$.

\section{References}

1 Williams MA, McAllister JP, Walker ML, et al. Priorities for hydrocephalus research: report from a National Institutes of Healthsponsored workshop. J Neurosurg 2007;107(5, Suppl):345-357
2 Bassan $\mathrm{H}$. Intracranial hemorrhage in the preterm infant: understanding it, preventing it. Clin Perinatol 2009;36: 737-762, v

3 van Ravenswaaij-Arts CM, Kollée LA, Hopman JC, Stoelinga GB, van Geijn HP. Heart rate variability. Ann Intern Med 1993;118: 436-447

4 de Beer NAM, Andriessen P, Berendsen RCM, Oei SG, Wijn PF, Oetomo SB. Customized spectral band analysis compared with conventional Fourier analysis of heart rate variability in neonates. Physiol Meas 2004;25:1385-1395

5 Wolfberg AJ, Derosier DJ, Roberts T, et al. A comparison of subjective and mathematical estimations of fetal heart rate variability. J Matern Fetal Neonatal Med 2008;21:101-104

6 Olischar M, Klebermass K, Kuhle S, Hulek M, Messerschmidt A, Weninger M. Progressive posthemorrhagic hydrocephalus leads to changes of amplitude-integrated EEG activity in preterm infants. Childs Nerv Syst 2004;20:41-45

7 Javorka K, Buchanec J, Kellerova E. Circulation in Fetuses, Newborns, Children and Adolescents. Regulation and Disorders. Martin: Osveta; 2009

8 Robles P, Poblano A, Hernández G, Ibarra J, Guzmán I, Sosa J. Cortical, brainstem and autonomic nervous system dysfunction in infants with post-hemorrhagic hydrocephalus. Rev Invest Clin 2002;54:133-138

9 Longin E, Gerstner T, Schaible T, Lenz T, König S. Maturation of the autonomic nervous system: differences in heart rate variability in premature vs. term infants. J Perinat Med 2006;34: 303-308

10 Krueger C, van Oostrom JH, Shuster J. A longitudinal description of heart rate variability in 28-34-week-old preterm infants. Biol Res Nurs 2010;11:261-268 